\title{
Sculpture et datation, le renouveau d'un enjeu
}

Dating sculptures: new developments

\section{Christian Sapin}

\section{OpenEdition \\ Journals}

Édition électronique

URL : https://journals.openedition.org/cel/18687

DOI : $10.4000 /$ cel. 18687

ISSN : 2262-208X

Éditeur

École du Louvre

\section{Référence électronique}

Christian Sapin, «Sculpture et datation, le renouveau d'un enjeu », Les Cahiers de l'École du Louvre [En ligne], 17 | 2021, mis en ligne le 16 novembre 2021, consulté le 13 décembre 2021. URL : http:// journals.openedition.org/cel/18687 ; DOI : https://doi.org/10.4000/cel.18687

Ce document a été généré automatiquement le 13 décembre 2021.

\section{(c) $(1) \odot$}

Les Cahiers de l'École du Louvre sont mis à disposition selon les termes de la licence Creative Commons Attribution - Pas d'Utilisation Commerciale - Pas de Modification 4.0 International. 


\title{
Sculpture et datation, le renouveau d'un enjeu
}

\author{
Dating sculptures: new developments
}

Christian Sapin

1 Entre archéologie et histoire de l'art, la question de datation des sculptures dans la plupart des travaux a souvent été abordée à partir d'un seul critère: sources historiques du site de provenance, comparaison formelle, ou morphologie. Cette question de la datation est générale et chacun ici connaît ce problème qui a été particulièrement mis en exergue par Jean Wirth dans La datation de la sculpture médiévale ${ }^{1}$, autocritique de l'histoire de l'art et de ses méthodes visant surtout le Moyen Âge central. Le haut Moyen Âge qui nous intéresse ici, tout en recouvrant les mêmes questions, pose de manière encore plus forte celle des remplois, et par là même celle de la création d'œuvres dans une société où la demande est moins forte qu'elle ne sera au Moyen Âge des XIII ${ }^{e}-X^{e}$ siècles. Pour cette période haute où les textes sont quasi absents pour désigner l'œuvre, les approches méthodologiques doivent être d'autant plus renouvelées pour les études et les analyses.

2 Il me semble que tout discours n'a de sens que si l'objet considéré appartient réellement à un contexte reconnu, et non placé en fonction des modes, des a priori ou de constructions théoriques. C'est ainsi que je suis depuis longtemps avec d'autres chercheurs préoccupés par ces questions de datation de référence pour les premières sculptures carolingiennes ou romanes. C'est pour mieux dater les chapiteaux de SaintPierre-l'Estrier d'Autun (Saône-et-Loire), dont les périodes proposées pour leur réalisation pouvaient s'étaler sur deux siècles, que j'ai entrepris des fouilles archéologiques sur le site en $1976^{2}$. C'est également pour mieux situer un ensemble important de décor de stuc découvert à Vouneuil-sous-Biard que j'ai repris la documentation de fouille et proposé des analyses archéométriques qui allaient décaler de trois siècles leur datation ${ }^{3}$. 


\section{Quels enjeux pour le haut Moyen Âge?}

3 L'enjeu évoqué en titre est à la fois celui de la sculpture, tel que l'on vient de l'évoquer en introduction avec deux exemples, mais c'est aussi celui de l'architecture à laquelle le décor sculpté se rattache, aussi bien dans son élévation qu'à travers son mobilier liturgique originel ou rapporté. Trois autres sites bien connus parmi les plus anciens de Gaule illustrent parfaitement cette problématique : la crypte de Jouarre, le baptistère de Poitiers, la crypte de Saint-Laurent de Grenoble, et leur décor sculpté. Leur datation reposait en grande partie, rappelons-le, autant sur les seules sources historiques, pour les deux premiers, que sur des a priori relevant d'un chauvinisme national ou local. Ces approches parfois étroites traduisaient les errances dans lesquelles se trouvaient les travaux sur un haut Moyen Âge manquant de certitudes sur lesquelles s'appuyer et conduisant souvent à des manipulations de chronologie. Ces dernières ont été dénoncées sans nuances par Jean Wirth dans son livre. Pour ne pas dépendre des seules analyses stylistiques, il y prône avec raison une analyse critique des sources et des études archéologiques ou de l'archéométrie ${ }^{4}$. Cependant pour les exemples les plus anciens, l'auteur se prend lui-même à son propre piège en mettant en avant la datation proposée par Jean Hubert pour Jouarre, qu'il rappelle encore dans sa conclusion. Compte tenu des évolutions méthodologiques, on est en droit de s'interroger sur plusieurs de ces sites et sur les méthodes de datation désormais proposées.

\section{Datation et chronologie relative}

4 Deux exemples de chapiteaux trouvés lors de fouilles en Suisse illustrent bien l'importance parfois déterminante d'une chronologie relative. C'est le cas du chapiteau réemployé dans un des états du baptistère du groupe épiscopal de Genève, fouillé par Charles Bonnet ${ }^{5}$. La chronologie des faits et le mobilier associé permettent de proposer une fourchette réduite. En effet, ce chapiteau (b 163), étudié par Guido Faccani ${ }^{6}$, peut appartenir au $2^{\mathrm{e}}$ état du baptistère (après 400 ), puisqu'il a été trouvé réemployé en fondation du ciborium du $3^{\mathrm{e}}$ baptistère du début du $\mathrm{VI}^{\mathrm{e}}$ siècle (fig. 1). 
Fig. 1. Chapiteau remployé dans la cathédrale de Genève

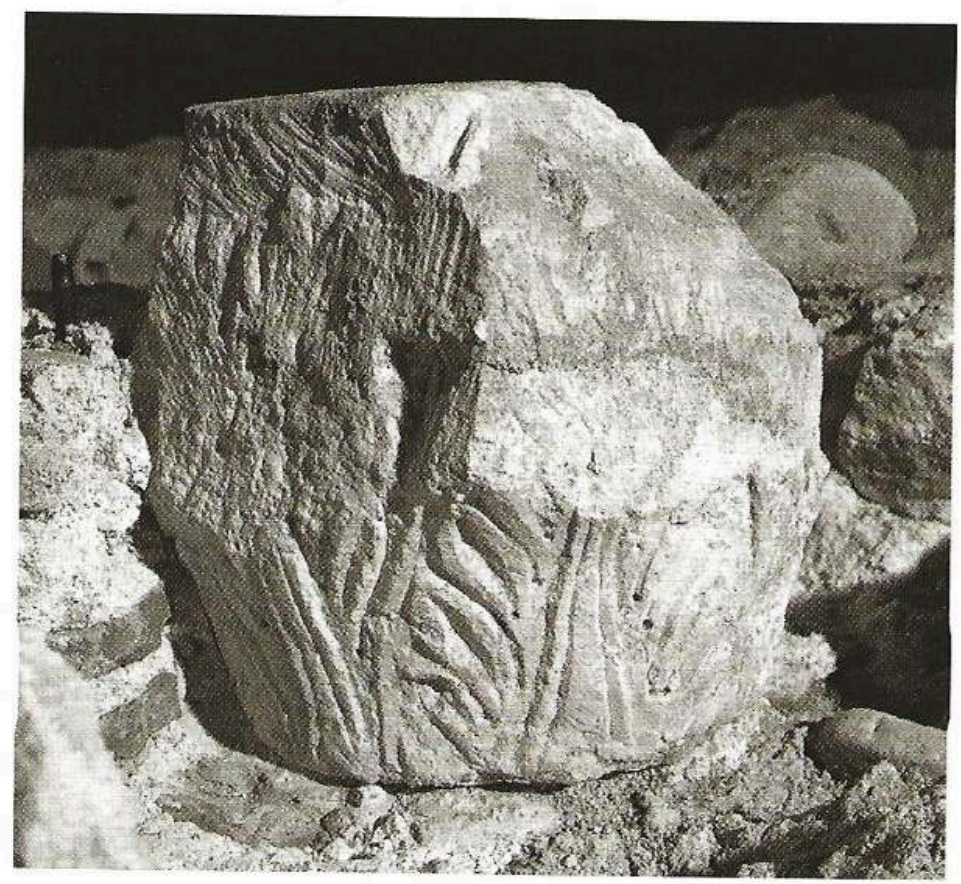

Chapiteau retrouvé remployé dans le baptistère du Vle siècle lors des fouilles de la cathédrale, Genève (Suisse).

(c) Service archéologique cantonal de Genève

Dans le cas du chapiteau réutilisé dans le seuil de fondation du clocher (XVIII siècle) de l'église de Meinier (Suisse), l'écart est cette fois trop grand pour soutenir une datation précise. C'est le lien établi avec les résultats de la fouille de l'église elle-même ${ }^{7}$, qui peut permettre de proposer pour ce chapiteau une contemporanéité dans le site aux $\mathrm{V}^{\mathrm{e}}$ $\mathrm{VI}^{\mathrm{e}}$ siècles. Par ailleurs, les rapprochements formels avec des chapiteaux de Saint-Alyre de Clermont ou de Saint-Germain-des-Prés à Paris, viennent conforter cette hypothèse. Un autre exemple encore plus explicite est la découverte faite à Brioude (Haute-Loire) lors des fouilles du baptistère ${ }^{8}$. Ici, la chronologie relative et encore une fois la stratigraphie générale du site permettent de proposer une datation antérieure à la couche stratigraphique du VII siècle et à son mobilier (fig. 2). On ne peut exclure une réutilisation d'une construction plus haute que le siècle précédent et, compte tenu de certains critères stylistiques, on peut admettre comme fourchette pour ce chapiteau en arkose, le $\mathrm{V}^{\mathrm{e}}$-début du $\mathrm{VI}^{\mathrm{e}}$ siècle. D'autres exemples issus de l'archéologie de sites de l'Antiquité tardive pourraient être mentionnés : ainsi ceux des villae du Sud-Ouest de la France dont les résultats ont été mis en évidence dans les années $1980-1990^{\circ}$, pour les conséquences dans la datation des sculptures proposées par plusieurs chercheurs, dont Jean Cabanot ${ }^{10}$. 
Fig. 2. Chapiteau de l'ancien baptistère de Brioude

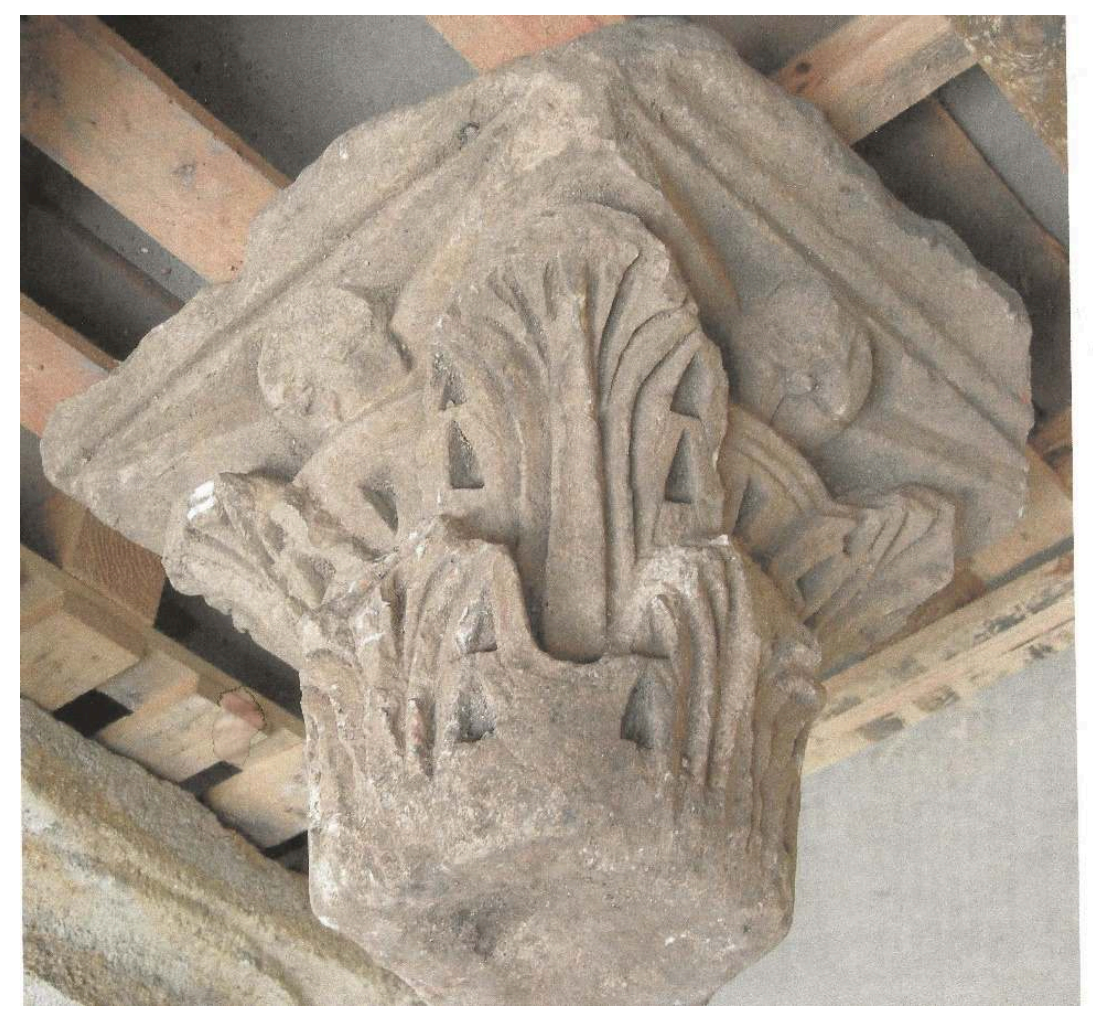

Fouilles de l'ancien baptistère, Brioude (Haute-Loire).

(c) F. Gauthier

\section{Datation et étude du monument}

6 La datation grâce à l'étude du monument suffit-elle ? La question a pu être plusieurs fois posée. Ce fut le cas pour Saint-Laurent de Grenoble qui possède dans le décor de la crypte un nombre important de chapiteaux: ensemble homogène ou pas. Renée Colardelle ${ }^{11}$ fait état des discussions variées issues des interprétations de Jean Hubert ${ }^{12}$ ou d'élisabeth Chatel $^{13}$, à partir des critères stylistiques et situant les sculptures entre le $\mathrm{VI}^{\mathrm{e}}$ et le $\mathrm{XI}^{\mathrm{e}}$ siècle selon les comparaisons retenues. Certains choix aboutissent ainsi curieusement à des datations pouvant relever, comme en d'autres domaines, d'une "moyenne ", en l'occurrence ici autour du VIII e siècle. Les arguments des uns et des autres dépendaient des références comparatives prises en compte. Jean Hubert préférait ainsi rajeunir l'édifice au milieu du VIII siècle, en se fondant sur la similitude du plan avec celui de la crypte de Saint-Pothin à Saint-Nizier de Lyon, sur celle de la colonnade avec celle de Germigny ou sur la typologie des formes de canthare ${ }^{14}$. L'étude archéologique du site et de son bâti permet de situer désormais la construction et le

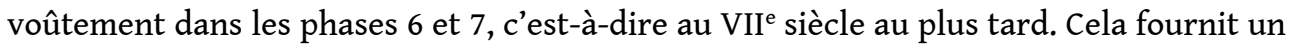
terminus antequem, mais n'interdit pas la possibilité d'une étude encore à faire chapiteau par chapiteau qui permettrait de déceler plus précisément des remplois ou des ajouts postérieurs à cette date, même si une telle intervention n'est pas évidente.

7 C'est également pour cette même période du haut Moyen Âge que l'étude d'ensemble du baptistère de Poitiers, revu par Brigitte Boissavit-Camus ${ }^{15}$, aboutit à un phasage plus clair des étapes de la construction, et ici plus évident qu'à Grenoble pour les décors 
sculptés pouvant s'y rattacher, étudiés par Anne Flammin ${ }^{16}$. L'ensemble se situerait dans un état 5 , daté par l'archéométrie du $3^{\mathrm{e}}$ quart du VII ${ }^{\mathrm{e}}$ siècle. Cette nouvelle datation est finalement proche de certaines propositions anciennes ${ }^{17}$, qui reposaient sur une première approche globale du bâti, des fouilles et sur les dates de l'épiscopat d'Ansoald (av. 677-fin VII ${ }^{\mathrm{e}}$ siècle) ; c'est-à-dire nettement après la date du VI $\mathrm{I}^{\mathrm{e}}$ siècle proposée par François Eygun, contestant le Père de La Croix et Jean Hubert ${ }^{18}$. Certains chapiteaux de marbre en partie inférieure sont à l'évidence des réemplois importés, probablement prélevés sur des villae ou venant de l'état précédent. Tandis qu'à l'étage des fenêtres, le décor de chapiteaux peut être jugé contemporain de cet état $5 \mathrm{du}$ monument ainsi que les reliefs extérieurs ${ }^{19}$.

\section{La sculpture sans monument}

Qu'en est-il des œuvres nombreuses conservées dans les musées et dépôts lapidaires, trouvées hors du contexte stratigraphique, même dans des monuments connus et étudiés, ou encore issues de collections anciennes non documentées ? Observons ainsi quelques exemples de Bourgogne, comme ces trois chapiteaux du musée d'Alise (Côted'Or), visiblement œuvres ou bases antiques retaillées (fig. 3).

Fig. 3. Chapiteau de la basilique d'Alise

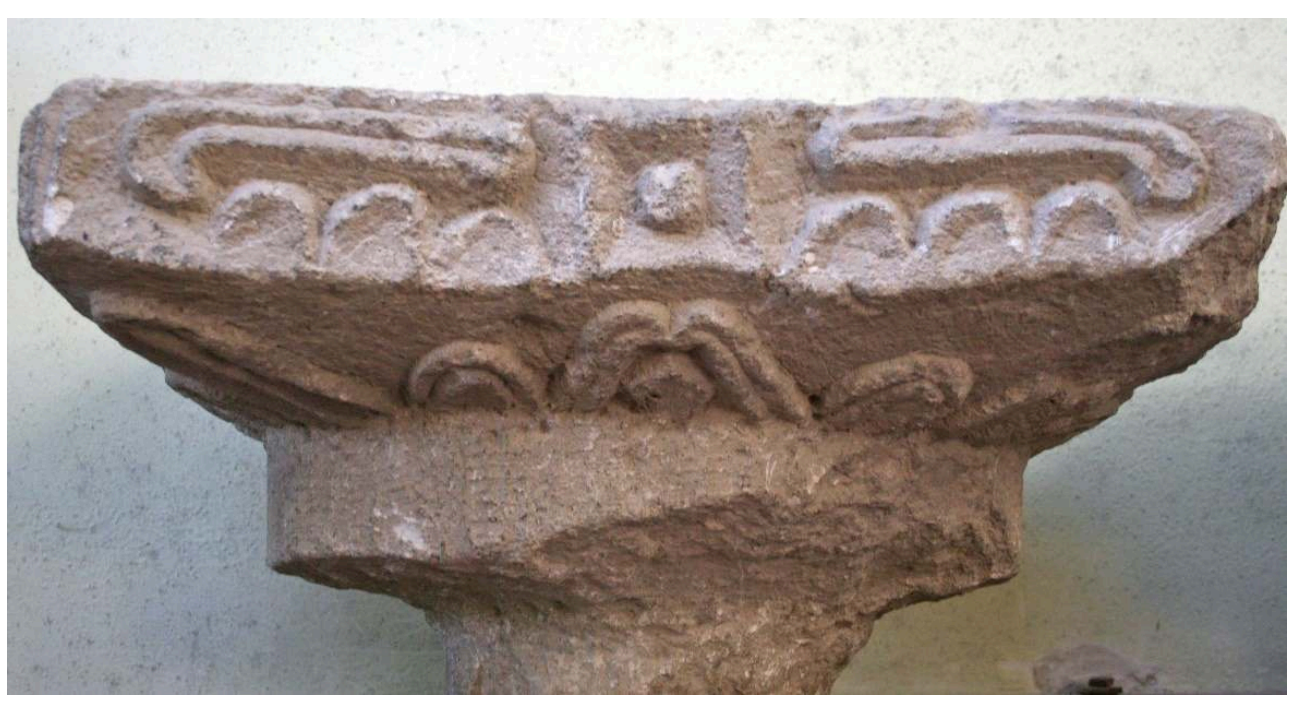

Chapiteau provenant des fouilles de la basilique, Alise-Sainte-Reine (Côte-d'Or), Musée d'Alise.

(c) Cliché Claude Grapin. Musée Alésia, Conseil départemental de la Côte-d'Or

On serait tenté, avec le conservateur du musée ${ }^{20}$, de rapprocher ces chapiteaux trouvés à proximité de la basilique chrétienne du décor supposé de celle-ci et plus précisément encore d'une valorisation de l'ancien mausolée devenu chœur de l'église, comme l'a montré Patrice Wahlen ${ }^{21}$. Ceci a pu se produire à la fin du haut Moyen Âge, soit peu avant le départ des reliques de sainte Reine pour le monastère de Flavigny, soit peu après, afin de garder le souvenir du lieu saint. C'est du moins la question qui se pose à la vue de ces chapiteaux atypiques, dont les seuls éléments formels autorisant des rapprochements sont les languettes sur l'abaque qui pourraient être rapprochées des figurations à deux brins des piliers carolingiens de Flavigny. 
D'autres œuvres sans filiations imposent des rapprochements formels. Voyons le cas du chapiteau n ${ }^{\circ} 101$ du musée de Chalon-sur-Saône, qu'un ancien inventaire donne comme un don d'un certain J. Bessy en 1862 et provenant, avec d'autres œuvres, de Cluny, sans préciser s'il s'agit du site ou d'une collection privée ${ }^{22}$. Le chapiteau de marbre de grandes dimensions (hauteur $0,45 \mathrm{~m}$; largeur $0,50 \mathrm{~m}$ ) est typique dans la découpe de ses feuilles et de ses aplats intermédiaires de chapiteaux des $\mathrm{V}^{\mathrm{e}}-\mathrm{VI}^{\mathrm{e}}$ siècles conservés à Saint-Victor de Marseille. On pourrait évoquer également un chapiteau en marbre d'une collection privée, acheté à Paris et qui selon le vendeur viendrait d'Orléans (Loiret) (fig. 4).Certains détails comme les hélices plates et le dé au motif circulaire avec croix à deux brins le rapprochent curieusement d'un chapiteau issu aussi d'Orléans et attribué à l'ancienne église Saint-Paul par Jean Hubert et Pierre Rousseau mais les interrogations quant à sa provenance restent tout de même nombreuses ${ }^{23}$. Dans le désir légitime de rattacher une œuvre à un site ou à un monument, citons les exemples d'œuvres aujourd'hui au musée de Sens (Yonne) comme le chapiteau provenant de Saint-Didier ${ }^{24}$. Dans la plupart des cas, on constate que la connaissance archéologique des sites est très faible pour apprécier une argumentation quant à la datation d'un objet pouvant en provenir.

Fig. 4. Chapiteau en marbre

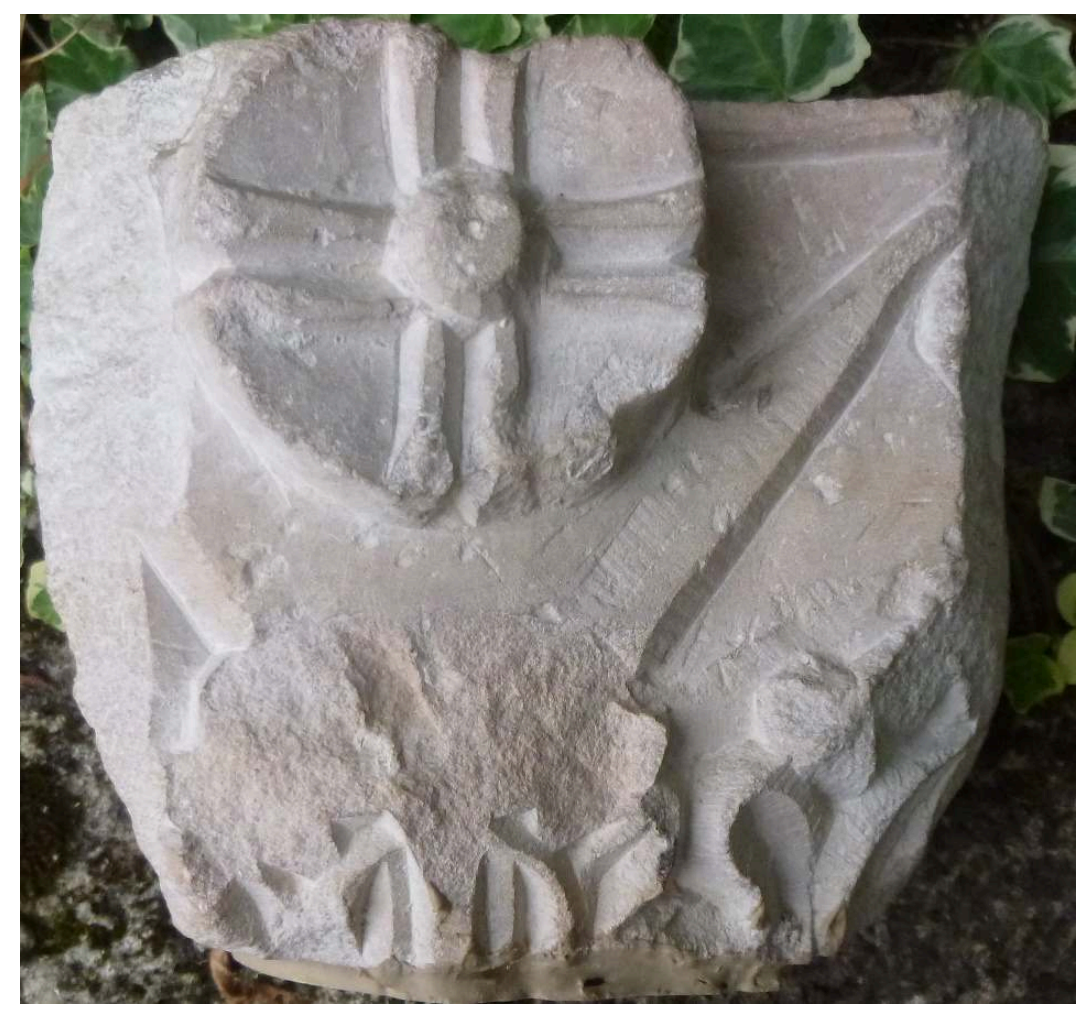

Chapiteau sans provenance connue, coll. privée, Autun.

(C) Christian Sapin

D'autres exemples, cette fois hors de Bourgogne, restent complexes comme ce chapiteau découvert sur un site de Charente-Maritime près de Sablonceaux en 1981 (fouille Christian Vernou) et qui pourrait résulter des transformations tardives d'une villa antique. Par ailleurs, il faut rappeler que l'étude du contexte archéologique ou architectural ne suffit pas toujours. On peut pour cela évoquer le cas des nombreux 
stucs découverts en remblais dans une chapelle du prieuré de Vouneuil-sous-Biard près de Poitiers. L'intérêt du stuc comme matériau est son adéquation à la structure et son impossible remploi. Leur présence encore en élévation est ainsi assez exceptionnelle, comme on le constate encore à Saint-Victor de Marseille ou plus encore au baptistère des Orthodoxes à Ravenne ${ }^{25}$. Ainsi, on aurait pu penser que les stucs trouvés dans une couche de remblais au sein d'une chapelle provenaient du décor de celle-ci ou du monument qui l'avait précédé et dont on voyait encore les murs. L'archéologie du bâti a démontré qu'en réalité, ces maçonneries étaient postérieures et n'avaient pas de liens avec le décor sculpté trouvé en fouille ${ }^{26}$. Dans cet exemple, seule l'archéométrie des ossements servant de tenons lors de la fabrication des stucs a permis une datation. Celle-ci s'éloigne de celle issue des rapprochements faits un premier temps avec des stucs plus connus, en particulier ceux de Disentis (Suisse), datés de l'époque carolingienne ${ }^{27}$. La nouvelle date proposée entre la fin $d u \mathrm{~V}^{e}$ et le début du $\mathrm{VI}^{\mathrm{e}}$ siècle renvoyait à un autre univers, non carolingien mais rattaché à l'Antiquité tardive, celui des stucs de Ravenne. Les rapprochements formels et la grammaire décorative devenaient alors beaucoup plus évidents qu'avec Disentis. Derrière ces datations nouvelles, c'est une autre conception du décor, souvent disparu et contemporain de celui en pierre, qui s'est imposée, exigeant un autre regard sur ces périodes.

\section{Sculpture et monuments carolingiens}

12 Finalement on comprend que la datation par l'étude du monument ou du contexte archéologique ne suffit pas toujours. Il faut un croisement de plusieurs approches relevant autant de l'histoire de l'art que de l'archéométrie, comme en témoignent certaines avancées de la recherche de ces vingt dernières années. On peut regarder ce qu'il en est pour l'époque carolingienne. Ayant souvent à l'esprit le décor du mobilier liturgique des chancels, très présent dans les musées notamment italiens, on pourrait penser que cette période est mieux connue que celle qui la précède. En réalité, la perception du décor architectural originel de ces édifices n'est pas si aisée à saisir et les datations ont longtemps été fantaisistes, induites parfois seulement par les sources textuelles. Il existe cependant des constructions encore en élévation, avec des sculptures en place comme Saint-Justin à Höchst, Corvey, Aix-la-Chapelle ou encore Germigny-des-Prés (Loiret), qui peuvent servir de repères. Dans plusieurs des cas se pose le problème des restaurations anciennes ou récentes, mais également dès l'origine. Ainsi, comment comprendre la contemporanéité de décors carolingiens entre réemplois, imitations et créations dans la crypte de Saint-Germain d'Auxerre ? On peut voir dans la confession de la crypte quatre chapiteaux: deux à l'est récemment réexaminés ${ }^{28}$, dont la partie inférieure de la corbeille a été retaillée en supprimant le premier rang de feuilles (fig. 5). Il pourrait s'agir de remplois d'œuvres antérieures ou d'une adaptation de chapiteaux prévus pour l'église supérieure au format des colonnes de la crypte et à son voûtement ${ }^{29}$. Un chapiteau présentant le même décor de feuilles avec suppression du rang inférieur existe dans les collections du musée ${ }^{30}$. Dans la confession, les deux autres chapiteaux sont totalement différents : celui du sud-ouest avec ses palmettes retournées aux angles est une œuvre originelle proche du décor des chapiteaux peints en trompe-l'œil, tandis que l'autre est un faux chapiteau réalisé en stuc et couvrant le haut de la colonne antique remployée. On constate ainsi une diversité d'attitude pour le décor d'un même lieu. Cette variété pourrait être la conséquence non d'une simple économie de matériau, mais de l'affirmation de la 
légitimité et de l'ancienneté du lieu de l'inhumation du saint. Ceci nous permet de dire que sans la connaissance de l'emplacement des œuvres dans l'espace originel, on peut se méprendre sur le sens du remploi et sur la datation des œuvres réalisées en fonction de celui-ci.

Fig. 5. Chapiteau de la confession

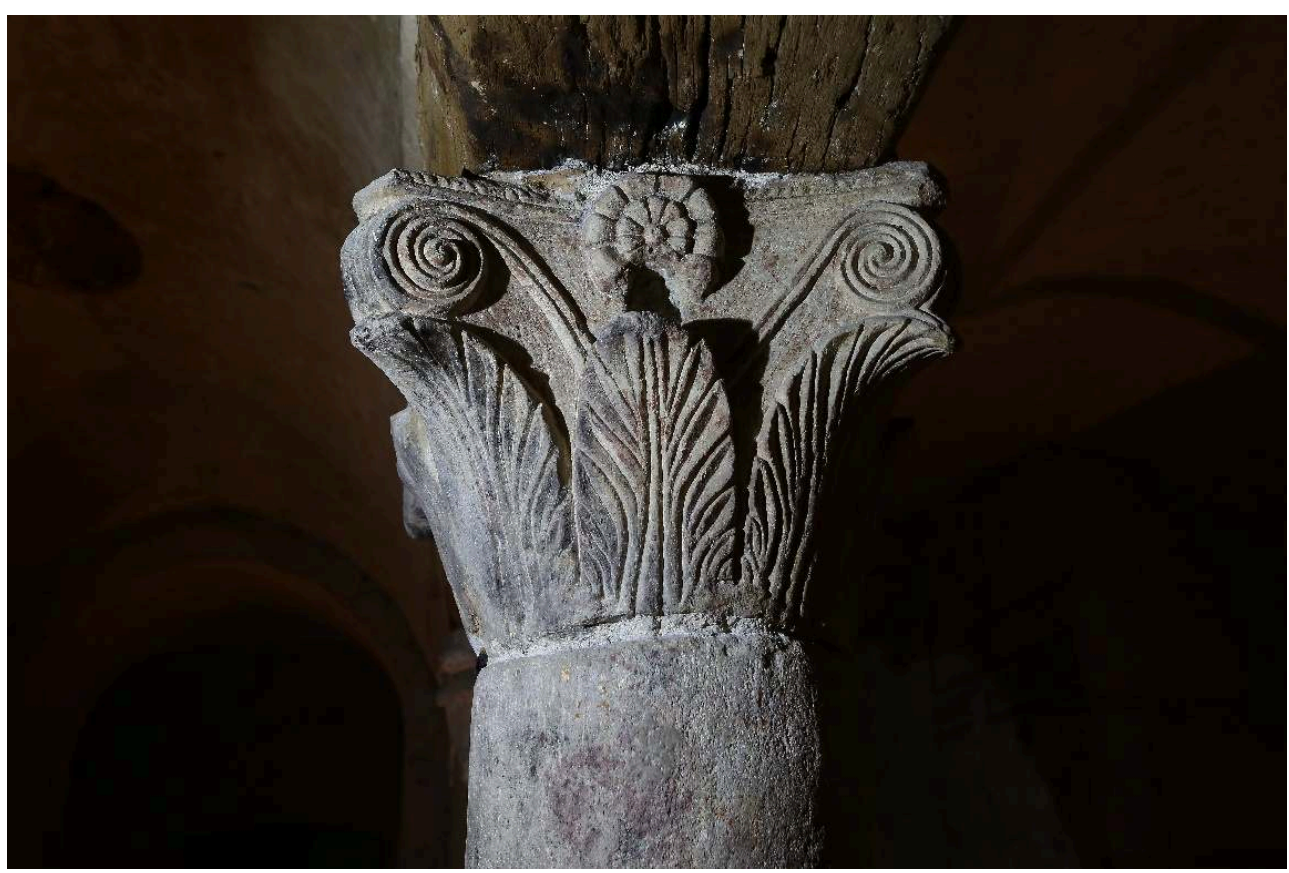

Chapiteau du nord-est de la confession, Crypte, Auxerre (Yonne).

(c) M. Glaus

Le site abbatial de Saint-Gall (Suisse), fouillé par Hans Rudolf Sennhauser et dont les sculptures viennent d'être étudiées par Guido Faccani, illustre parfaitement cette diversité des possibles pour la position des sculptures pouvant provenir d'un même atelier au sein d'un même site. Le travail de recherches comparatives avec d'autres sculptures demande comme on le sait attention et distance ${ }^{31}$. Toujours pour cette période, on ne peut que renvoyer au colloque de Metz sur la question des chancels, en particulier celui de Saint-Pierre aux Nonnains, qui soulève parfaitement le problème des limites du raisonnement entre données in situ et comparaisons, ouvrant à des datations étalées selon les auteurs sur deux siècles ${ }^{32}$. Il faut aussi poser la question du mobilier liturgique qui par essence n'est pas établi avec le bâti et n'est donc pas nécessairement contemporain des dates attribuées à la construction. C'est ainsi que le pilier à entrelacs conservé comme support dans les cryptes de Flavigny (Côte-d'Or) perd sa valeur d'argument datant pour la construction, depuis que l'on a retrouvé d'autres piliers dont l'attribution aux seules cryptes n'est pas attestée. Le fameux pilier carolingien pourrait être un réemploi utilisé dans la reprise du couloir au $\mathrm{XI}^{\mathrm{e}}$ siècle et venant d'une partie carolingienne disparue, comme le cloître ${ }^{33}$.

14 Toujours avant l'an Mil, les questions restent encore nombreuses, faute souvent d'études complètes du bâti. En Normandie par exemple, le paysage du décor sculpté s'est éclairci à Saint-Pierre de Jumièges. Les chapiteaux de sa tribune ont longtemps été donnés comme réalisés au $\mathrm{X}^{\mathrm{e}}$ siècle, si ce n'est au $\mathrm{XI}^{\mathrm{e}}$ siècle. Cette attitude résultait essentiellement d'une interprétation erronée des sources qui imaginaient une 
reconstruction importante seulement après le passage des Vikings ${ }^{34}$. Depuis, étude de bâti et archéométrie ont montré que l'on était autour des années 800 et à regarder de près, force était de reconnaitre que ce type de chapiteau s'accordait parfaitement avec ceux connus pour le IX ${ }^{\mathrm{e}}$ siècle (fig. 6). D'autres enjeux entre monuments et sources attendent de telles reprises d'étude. C'est le cas à Chambéry (Savoie), où l'église de Lemenc, n'en finit pas de questionner, sans arguments décisifs quant à la datation des chapiteaux de sa rotonde. On n'échappe pas au jeu formel. Certains ont voulu y voir des chapiteaux carolingiens essentiellement à partir des sources précisant que la villa de Lemenc était donnée en 866 à Thiberge, épouse de Lothaire II, roi de Lotharingie. Il est vrai que par certains aspects la tradition carolingienne n'est pas loin, si l'on considère l'abaque à double filet et les feuilles lisses aux nervures marquées. Mais les crochets sont plus tardifs et éloignés de chapiteaux comme ceux de Lorsch, désormais datés vers 900.

Fig. 6. Chapiteau de tribune, en place

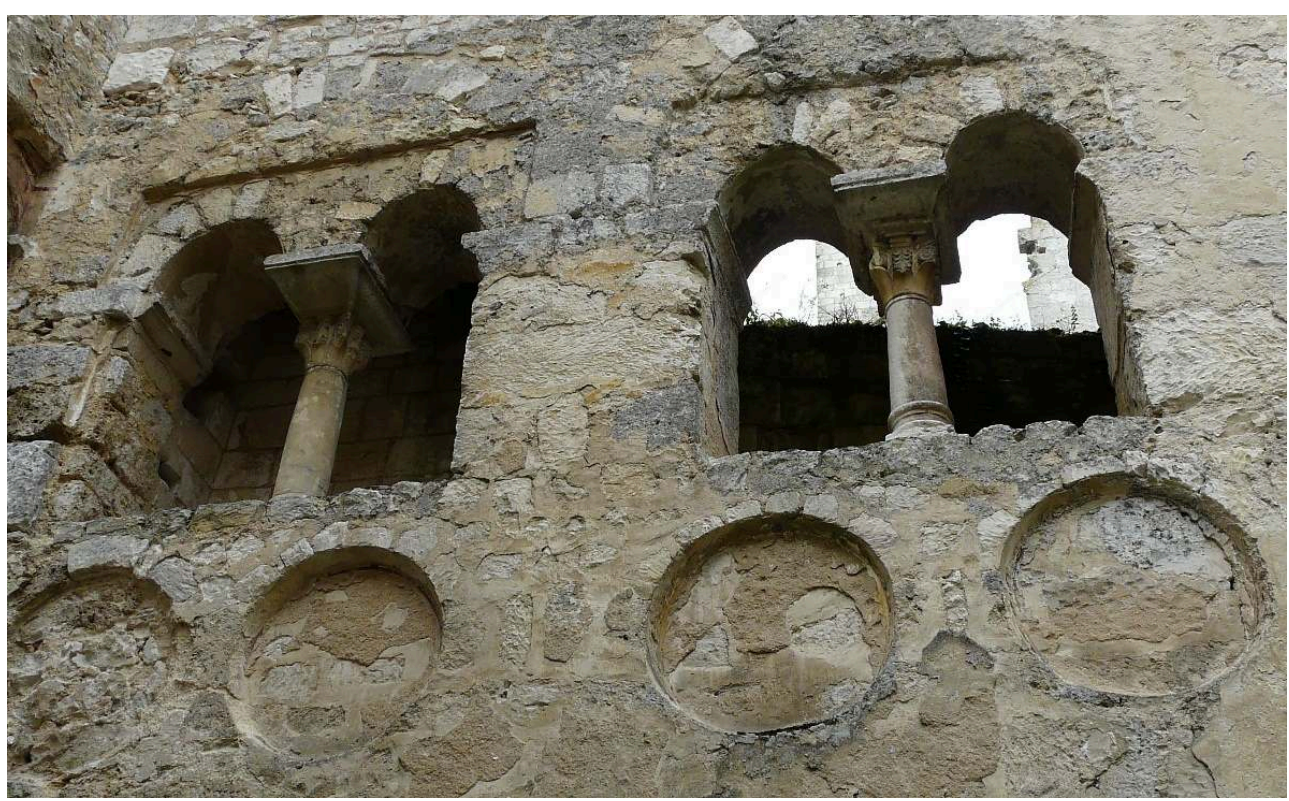

Chapiteau en place, tribune nord, Jumièges (Seine Maritime).

(c) F. Héber-Suffrin

\section{Sculpture romane et construction}

Une différence d'approche s'impose pour la sculpture romane après l'an Mil, du fait de la multiplication des œuvres réalisées et surtout conservées. L'étude récente du bâti de nombreux édifices de cette période a-t-elle été pour autant le gage d'une datation plus précise de la sculpture de ces monuments ou au contraire, par l'archéométrie par exemple, a-t-elle abouti à une remise en cause des anciennes propositions? On connaissait pour cette période les avancées considérables faites en particulier par les travaux de Louis Grodecki, Marcel Durliat ou Éliane Vergnolle. À la fin du XXe siècle, de grandes lignes ont commencé alors à se dessiner pour saisir la mise en place des ensembles sculptés. On accepte ainsi aujourd'hui en plusieurs régions l'idée d'émergence d'ateliers et donc du rattachement à ceux-ci d'autres ensembles pour datation. C'est le cas en région centre avec Saint-Benoît-sur-Loire (Loiret), ou en 
Bourgogne avec les sites monastiques de Saint-Pierre de Flavigny, d'Autun avec SaintPierre-l'Estrier (Saône-et-Loire) ${ }^{35}$, ou de Saint-Philibert de Tournus. En dépit d'une grande différence dans l'expression plastique de ces ateliers (sans parler de l'existence de plusieurs mains), il est possible aujourd'hui d'accepter pour leur exécution des datations proches, entre 1010-1025, et par là même l'idée de contemporanéité pour des créations différentes en d'autres régions.

Malgré ces regroupements possibles en atelier, les questions d'identification et de datation demeurent, pour des œuvres trouvées directement in situ ou en relation avec des sites connus. On le voit à Cluny, non pour la grande église de Cluny III où les doutes sont seulement vis-à-vis des emplacements, mais pour des chapiteaux attribués par $\mathrm{K}$. J. Conant à Cluny II. On retient pour l'instant cette hypothèse mais on aurait pu attendre que les fouilles récentes (2006-2013) nous éclairent sur le décor de cette église de l'an Mil. Au moins par le lieu de leur découverte ${ }^{36}$ et par leurs dimensions, on est en droit d'attribuer d'autres œuvres de morphologies différentes au mobilier liturgique de la seconde église, c'est-à-dire entre 960 et 1030 - en acceptant que ce mobilier ait pu être renouvelé durant ces décennies. Un dernier exemple avec Saint-Martin d'Angers illustre cette période charnière avec des questions identiques entre chronologie relative et comparaisons. Sur ce site, plusieurs chapiteaux ont été trouvés, soit lors de fouilles archéologiques ${ }^{37}$, dans des remblais qui peuvent assurer une datation par stratigraphie précise, soit remployés dans la reconstruction de la croisée établie sous Foulques Nerra (987-1040). Pour ceux-ci (fig. 7), on pourrait admettre qu'ils appartenaient à un premier état de la croisée qui est daté par archéométrie entre le IX ${ }^{\mathrm{e}}$ et le début $\mathrm{du} \mathrm{X}^{\mathrm{e}}$ siècle $^{38}$, tandis qu'une approche simplement comparative aurait tendance à les placer plus tardivement, vers le $\mathrm{XI}^{\mathrm{e}}$ siècle. 
Fig. 7. Chapiteau de l'église Saint-Martin

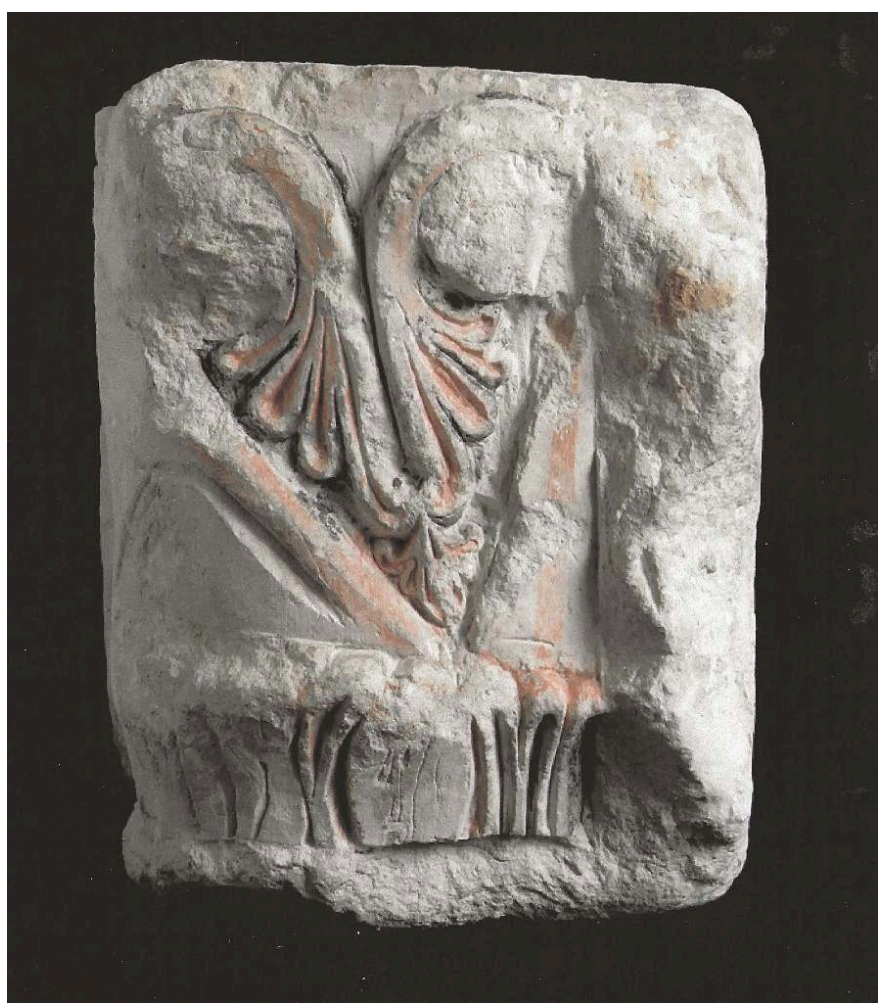

Chapiteau provenant de l'église Saint-Martin, Angers (Maine-et Loire).

(c) D. Prigent

17 Que ce soit pour comprendre et situer la sculpture elle-même ou bien pour l'architecture, ce sont autant de questions, souvent mêlées, qui demeurent et pour lesquelles, nous l'espérons, la future base de données du corpus en ligne apportera des réponses par l'interaction de critères croisés. C'est dans ce but que la présence dans les notices d'une rubrique sur les arguments de datation est essentielle. Ce besoin d'une fiabilité $\mathrm{du}$ raisonnement est nécessaire autant pour la sculpture que pour l'architecture, comme cela est demandé dans le protocole des fiches d'enregistrement de la base de données CARE-Corpus architecturae religiosae europeae IV-X saec. à l'échelle de l'Europe. C'est à cette condition que l'on acceptera, non pas un discours basé sur un progrès imaginaire des formes, mais sur l'existence d'une diversité possible de création dans un même moment de l'histoire. Penser ainsi la complexité et la diversité reste un programme toujours d'actualité.

\section{NOTES}

1. Jean Wirth, La datation de la sculpture médiévale, Genève, Droz, 2004.

2. Christian Sapin, «L'ancienne église de Saint-Pierre-l'Estrier à Autun », Archéologie Médiévale, XII, 1982, pp. 51-105. Ces premiers travaux ont entrainé l'étude plus globale de ce site remontant 
à l'Antiquité tardive. Voir la notice "Autun, Église Saint-Pierre-l'Estrier ", dans Les premiers monuments chrétiens de la France, t. 3, Paris, Picard, 1998, pp. 64-69.

3. C. Sapin - avec la collaboration de B. Palazzo-Bertholon, C. Treffort, «Les stucs de l'Antiquité tardive de Vouneuil-sous-Biard (Vienne) », 60e supp. , Gallia, Paris, CNRS éditions, 2009.

4. J. Wirth, op. cit. note 1, pp. 60 et suiv.

5. Charles Bonnet, Les fouilles de la cathédrale Saint-Pierre de Genève, les édifices chrétiens du groupe épiscopal, Genève, 2012.

6. Guido Faccani, «Sculpture architecturale provenant des fouilles de la cathédrale Saint-Pierre (IV ${ }^{\mathrm{e}}$ XII ${ }^{\mathrm{e}}$ siècle), dans C. Bonnet, op. cit. note 5, Genève, 2012, pp. 237-265.

7. Jean Terrier et alii, «Les fouilles archéologiques de l'église Saints-Pierre-et-Paul de Meinier ", Genava, nouv. sér., t. LII, 2004, pp. 215-310.

8. Chapiteau découvert lors des fouilles du baptistère de Brioude par Fabrice Gauthier (Inrap), que je remercie. Voir Laurent Arbaret, Fabrice Gauthier, «Les sarcophages du quartier du baptistère de Brioude: origine, importations et évolutions pendant l'époque mérovingienne ", Les sarcophages de l'Antiquité tardive et du haut Moyen Âge, supplément 34 d'Aquitania, pp. 401-410.

9. Catherine Balmelle, Les demeures aristocratiques d'Aquitaine. Société et culture de l'Antiquité tardive dans le Sud-Ouest de la Gaule, Bordeaux-Paris, Éditions Ausonius, 2001.

10. Jean Cabanot, «Les éléments de décor monumental antérieurs à l'époque romane conservés à Saint-Sever (Landes) », Cahiers archéologiques, t. 42, 1994, pp. 35-64 ; Jacques Lapart, " Chapiteaux de marbre antérieurs à l'époque romane dans le Gers: nouvelles découvertes ", Archéologie du Midi médiéval, t. 3, 1985. pp. 3-12; Anne-Bénédicte Mérel-Brandenburg, La sculpture de l'Antiquité tardive en Languedoc méditerranéen et Roussillon (IV $\left.{ }^{e}-V I I I^{e} s.\right)$, Thèse de l'Université de Paris Sorbonne-Paris 4, 2007.

11. Renée Colardelle, La ville et la mort, Saint-Laurent de Grenoble, 2000 ans de tradition funéraire, Turnhout, Brepols, 2008.

12. Jean Hubert, «La "crypte" de Saint-Laurent de Grenoble et l'art du sud-est de la Gaule au début de l'époque carolingienne", Arte del primo millenio. Atti del $\mathrm{II}^{\circ}$ Convegno per lo studio dell'arte del medioevo, Pavia, sett. 1950, Turin, 1953, pp. 327-334.

13. Élisabeth Chatel, Recueil général des monuments sculptés en France pendant le haut Moyen Âge (IV $X^{e}$ siècles), t. II, Isère, Savoie, Haute-Savoie, Paris, Ed CTHS, 1981.

14. Voir R. Colardelle, op. cit. note 11, p. 188 pour le rappel des arguments des uns et des autres et l'attitude de Carol Heitz pour qui « ces sculptures ne peuvent être postérieures au VII ${ }^{\mathrm{e}}$ siècle » et qui rapportait une conversation avec Jean Hubert qui « regrettait sa datation un peu trop tardive ».

15. Brigitte Boissavit-Camus, Le baptistère Saint-Jean de Poitiers. De l'édifice à l'histoire urbaine, Bibliothèque de l'Antiquité tardive, 26, Turnhout, Brepols, 2014.

16. Anne Flammin, "Le décor installé dans le baptistère de Poitiers au VII ${ }^{\mathrm{e}}$ siècle ", dans B. Boissavit-Camus, op. cit. note 15, pp. 359-399.

17. Denise Fossard, "Les chapiteaux en marbre du VII ${ }^{\mathrm{e}}$ siècle en Gaule, style et évolution ", Cahiers archéologiques, t. 2, 1947, pp. 69-85.

18. Francois Eygun, « Le baptistère de Poitiers », Gallia, t. XXII, 1964, nº 1, pp. 137-171. Parmi les arguments peu scientifiques de cet auteur, on trouve dans sa réponse à l'article de Jean Hubert («Réflexions sur les monuments mérovingiens de Poitiers», Bulletin de la Société des Antiquaires de l'Ouest, t. IX, 1968, pp. 399-406) la phrase suivante: «Placer le baptistère et l'hypogée au même temps nous paraissait gros, disons impossible du point de vue de l'histoire de l'Art".

19. A. Flammin, op. cit. note 16.

20. Je remercie Claude Grapin de son accueil et des informations fournies.

21. Patrice Wahlen, "La basilique Saint-Reine d'Alésia, Reine au Mont-Auxois ", Le culte et le pèlerinage de sainte Reine des origines à nos jours, sous la direction de P. Baudry et D. Julia, Dijon, 
1997, pp.61-80 ; C. Sapin et P. Wahlen, «Alise-Sainte-Reine. Basilique présumée de SainteReine », Les Premiers Monuments chrétiens de la France, t. I, Paris, 1998, pp. 49-54.

22. Société d'Histoire et d'Archéologie de Chalon, 2, 1936, p. 154.

23. Pierre Rousseau, «Un chapiteau trouvé en Août 1955 dans les ruines de l'église Saint-Paul d'Orléans, séance du 24 octobre 1956, Bulletin de la Société nationale des antiquaires de France, 1956 (éd. 1958), pp. 104-106.

24. J. Hubert, "Un chapiteau carolingien trouvé au faubourg de Saint-Didier à Sens », dans Bulletin de la Société nationale des antiquaires de France, 1954-1955, pp. 64-67 ; C. Sapin, La Bourgogne préromane, Paris, 1986, p. 205.

25. Voir Stucs et décors de la fin de l'Antiquité au Moyen Âge ( $V^{e}-\mathrm{XI}{ }^{e}$ siècles). Actes du colloque international Poitiers 16-19 sept. 2004, Bibliothèque de l'Antiquité tardive, 10, Turnhout, Brepols, 2007.

26. C. Sapin, op. cit. note 3.

27. Rapprochement qui paraissait logique, voir Marie-Thérèse Camus, «Les stucs de Vouneuilsous-Biard », Cahiers de civilisation médiévale, vol. 33, 1990, pp. 151-152.

28. C. Sapin, "Saint-Germain d'Auxerre et la sculpture carolingienne en Bourgogne", communication au Colloque de Saint-Gall (Suisse), Frühmittelalterkirche Bauskulptur, 14-16 juin 2018, non encore publiée.

29. Rappelons que la confession de la crypte carolingienne s'adapte à l'espace de l'ancien oratoire mausolée et que sa hauteur est contrainte par le rocher et la hauteur souhaitée pour le niveau du presbyterium supérieur.

30. C. Sapin, op. cit. note 24, p. 197.

31. Voir colloque de St Gall : Frühmittelalterkirche Bauskulptur, 14-16 juin 2018, non encore publié et Guido Faccani, Bauplastik des 1. Jahrtausends aus St.Gallen, Saint-Gall, 2021.

32. Le chancel de Saint-Pierre-aux-Nonnains, problématique complexe pour un ensemble exceptionnel, colloque international - Metz, 27-29 avril 2017, sous la direction d'Anne Adrian, Metz, Musée de la Cour d'Or - Metz Métropole, collection « Mémoires des réserves », 2, 2021.

33. C. Sapin, dans op. cit. note 34 et C. Sapin, «Disposition et construction monastique préromane et romane, la question des premiers cloîtres en Bourgogne ", De la Bourgogne à l'Orient. Mélanges offerts à Monsieur le Doyen Jean Richard, sous la direction de Jacques Meissonnier avec la collaboration de Christine Lamarre, Françoise Perrot, Alain Rauwel et Hugues Richard, Dijon, Académie des sciences, arts et belles-lettres de Dijon, Dijon, 2020, pp. 173-193.

34. Jacques Le Maho [Jim Morganstern col.], «Jumièges, église Saint-Pierre. Les vestiges préromans ", Congrès archéologique de France. Rouen et Pays de Caux, Paris, 2006, pp. 97-116.

35. C. Sapin, «Les chapiteaux de Saint-Pierre-L'Estrier à Autun (Saône et Loire, France) et les ateliers des débuts de la sculpture romane en Bourgogne », De la passion à la création. Hommage à Alain Erlande-Brandenburg, sous la direction de M. Jurkovic, Zagreb-Motovun, 2017, pp. 91-99.

36. Anne Baud, C. Sapin, Cluny, les origines du monastère et de ses églises, Paris, éd. du CTHS, 2019, pp. 189-191.

37. Daniel Prigent, «Les édifices antérieurs au milieu du XI ${ }^{\mathrm{e}}$ siècle en Val de Loire : l'évolution du regard de l'archéologue ", La mémoire des pierres, Bibliothèque d'Antiquité tardive, 29, Turnhout, Brepols, 2016, pp. 85-96.

38. D. Prigent, op. cit. note 37, p. 92 


\section{RÉSUMÉS}

Sur le modèle du corpus d'architecture $\mathrm{IV}^{\mathrm{e}}-\mathrm{X}^{\mathrm{e}}$ siècles, le corpus en préparation pour la sculpture de la même période en France intègre dans ses champs les divers arguments nécessaires à la datation des éléments sculptés. Entre archéologie et histoire de l'art, la datation des sculptures dans les travaux antérieurs a souvent été appréciée à partir d'un seul critère : sources historiques d'un site de provenance, comparaison formelle ou morphologie... L'estimation de l'ancienneté importante des exemples de chapiteaux à Jouarre, au baptistère de Poitiers ou à Saint-Laurent de Grenoble reposait en grande partie sur les sources historiques. Qu'en est-il aujourd'hui où l'archéologie a pu opérer sur ces mêmes sites? Comment comprendre la contemporanéité de décors carolingiens entre réemploi, imitations et créations comme à Saint-Germain d'Auxerre? Un renouveau a longtemps été situé pour la sculpture romane après l'an Mil. L'étude récente du bâti de nombreux édifices de cette période a-t-elle été un gage assurant à travers l'archéométrie une datation plus précise ou au contraire une remise en cause? Qu'en est-il des œuvres hors contexte de monuments connus ou étudiés? Autant de questions présentées avec des exemples et pour lesquelles, on l'espère, la base de données du corpus proposé fera apparaître des réponses par l'interaction de critères croisés.

Based on the corpus of fourth-tenth century architecture, the corpus for sculpture of the same period in France that is being prepared includes in its fields the various arguments necessary for the dating of sculpted elements. A combination of archaeology and art history, the dating of sculptures in earlier works was often assessed based on a single criterion, such as historical sources from the site of provenance, or formal or morphological comparisons. The estimation of the age of the capitals at Jouarre, the baptistery of Poitiers and Saint-Laurent de Grenoble was largely based on historical sources. How has this changed, now that archaeology has been able to assess these sites? How can we understand the contemporaneity of Carolingian decoration given the reused elements, replicas and new works, as at Saint-Germain d'Auxerre? It was long thought that the revival of Romanesque sculpture took place after the year 1000. Has the recent study of the frames of numerous buildings from this period been a guarantee of more precise dating through archaeometry or, on the contrary, a calling into question? What about works outside the context of known or studied monuments? All these questions are presented with examples and, we hope, the database of the proposed corpus will provide answers to them through the interaction of crossed criteria.

INDEX

Keywords : Early Middle Ages, Carolingian, Romanesque, capital, dating

Mots-clés : haut Moyen Âge, carolingien, roman, chapiteau, datation

\section{AUTEUR}

\section{CHRISTIAN SAPIN}

Christian Sapin est archéologue et historien de l'art. Directeur de recherche émérite au CNRS, Umr 6298 Artehis Dijon. Titulaire d'une thèse d'Histoire de l'art et d'archéologie à l'université de Paris Ouest-Nanterre et ancien élève à l'Ecole du Louvre. Outre des charges d'enseignement, il a assuré la responsabilité de plusieurs groupes de recherche notamment du GDR européen du CNRS 
sur les nouvelles méthodes de datation à partir des Terres cuites architecturales et a dirigé de nombreux chantiers archéologiques, en particulier sur le groupe épiscopal d'Autun (Saône-etLoire), l'ancienne abbaye Saint-Germain d'Auxerre (Yonne) ; Notre-Dame-sous-Terre du MontSaint-Michel (Manche), la crypte de Saint-Quentin (Aisne), l'abbaye de Cluny (Saône-et-Loire), le cloitre de Vézelay (Yonne).

The archaeologist and art historian Christian Sapin is emeritus director of research at the CNRS, UMR 6298 ARTEHIS Dijon. He has a PhD in art history and archaeology from the Université de Paris Ouest-Nanterre and is a graduate of the École du Louvre. In addition to his teaching duties, he oversees many research groups, notably the CNRS European research group on new methods of dating using architectural terracotta, and has directed many archaeological projects, including those involving the episcopal group of Autun, Saône-et-Loire; the former abbey of Saint-Germain d'Auxerre, Yonne; Notre-Dame-sous-Terre du Mont-Saint-Michel, Manche; the crypt of SaintQuentin, Aisne; the Abbaye de Cluny, Saône-et-Loire; and the cloister at Vézelay, Yonne. 